\title{
The perinatal postmortem examination ${ }^{1}$
}

\author{
F. A. LANGLEY \\ From the Department of Pathology, St Mary's Hospital for Women and Children, Manchester, and \\ the Department of Obstetrics and Gynaecology, Manchester University
}

SYNOPSIS In order to determine the cause of a perinatal death it is important to correlate the postmortem findings with the clinical histories of both mother and infant. The postmortem examination of a newborn infant differs from that of an adult in a number of ways, especially in the method of removal and dissection of the brain, the examination of the cervical spine, the dissection of the cardiovascular system for congenital abnormalities, and the examination of the placenta. A systematic technique for performing a perinatal necropsy is described. Stress is laid on measurement of the size of the infant and of the individual organs and comparison of these with known standards, allowance being made for the maturity of the infant.

A perinatal postmortem examination may be made for two purposes. First, to obtain as much information as possible to determine the immediate cause of death of a particular infant. Second, for a wider study along epidemiological lines to ascertain the part played by various factors in causing foetal and infant death. For both these purposes the mother's and infant's case notes should be available to the pathologist. Both naked-eye and histological examination of the tissue is necessary and in stillborn foetuses the placenta should always be examined; microbiological and serological studies may also be valuable.

'Copies of this paper may be obtained from the Publishing Manager, BMA House, Tavistock Square, London WC1H 9JR, price 20p each, the envelope marked, Journal of Clinical Pathology, BROADSHEETS.

\section{Weights and Measurements}

Since the size of the body and its organs varies with the maturity of the infant these must be measured accurately and compared with known standards. It is valueless and often misleading to state that an organ is enlarged without such a comparison.

The length of the body can best be measured using a graduated board with a fixed upright at its head and a movable upright at the feet (Figs. 1 and 2). When a board is not available the crown-heel length of the foetus can be measured by means of a thread (Fig. 3). The sitting height is approximately twothirds of the standing height. Bearing this relation in mind, a correction may be made to assess the expected length in such malformations as anencephaly

\begin{tabular}{|c|c|c|c|c|c|c|}
\hline \multirow{2}{*}{$\begin{array}{l}\text { Gestational } \\
\text { Age (wk) }\end{array}$} & \multirow{2}{*}{$\begin{array}{l}\text { No. of } \\
\text { Cases }\end{array}$} & \multicolumn{5}{|c|}{ Birth Weight $(k g)$} \\
\hline & & $+2 S D$ & $+1 S D$ & Mean & $-1 S D$ & $-2 S D$ \\
\hline $\begin{array}{c}32 \pm 1 \frac{1}{2} \\
34 \pm \frac{1}{8} \\
35 \pm \frac{1}{8} \\
36 \pm \frac{1}{8} \\
37 \pm \frac{1}{8} \\
38 \pm \frac{1}{8} \\
39 \pm \frac{1}{8} \\
40 \pm \frac{1}{8} \\
41 \pm \frac{1}{8} \\
42 \pm \frac{1}{8} \\
>42\end{array}$ & $\begin{array}{r}92 \\
68 \\
122 \\
238 \\
392 \\
719 \\
1629 \\
2784 \\
2597 \\
1518 \\
897\end{array}$ & $\begin{array}{l}3 \cdot 78 \\
3 \cdot 96 \\
4 \cdot 05 \\
4 \cdot 16 \\
4 \cdot 01 \\
4 \cdot 03 \\
4 \cdot 22 \\
4 \cdot 25 \\
4 \cdot 36 \\
4 \cdot 44 \\
4 \cdot 50\end{array}$ & $\begin{array}{l}3 \cdot 03 \\
3 \cdot 29 \\
3 \cdot 43 \\
3 \cdot 54 \\
3 \cdot 49 \\
3 \cdot 56 \\
3 \cdot 74 \\
3 \cdot 80 \\
3 \cdot 91 \\
3 \cdot 97 \\
3 \cdot 99\end{array}$ & $\begin{array}{l}2.28 \pm 0.75 \\
2.62 \pm 0.67 \\
2.81 \pm 0.62 \\
2.92 \pm 0.62 \\
2.97 \pm 0.52 \\
3.09 \pm 0.47 \\
3.26 \pm 0.48 \\
3.35 \pm 0.45 \\
3.46 \pm 0.45 \\
3.50 \pm 0.47 \\
3.48 \pm 0.51\end{array}$ & $\begin{array}{l}1 \cdot 53 \\
1.95 \\
2 \cdot 19 \\
2 \cdot 30 \\
2.45 \\
2.62 \\
2 \cdot 78 \\
2.90 \\
3.01 \\
3.03 \\
2.97\end{array}$ & $\begin{array}{l}0 \cdot 78 \\
1 \cdot 28 \\
1 \cdot 57 \\
1 \cdot 68 \\
1 \cdot 93 \\
2 \cdot 15 \\
2 \cdot 30 \\
2 \cdot 45 \\
2 \cdot 56 \\
2 \cdot 56 \\
2 \cdot 46\end{array}$ \\
\hline
\end{tabular}

Table Birth weights of single legitimate liveborn infants born in Newcastle 1960-62 (modified from G. Neligan, 1965) 


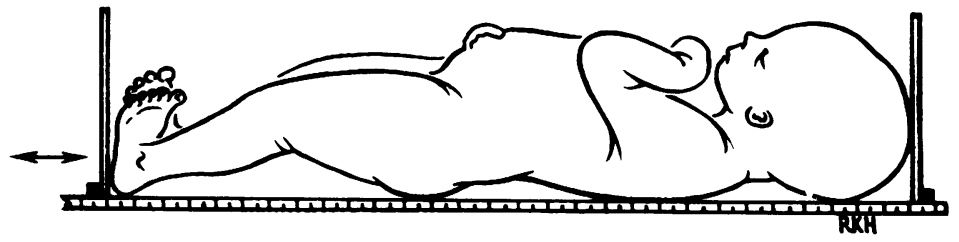

Fig. 1.

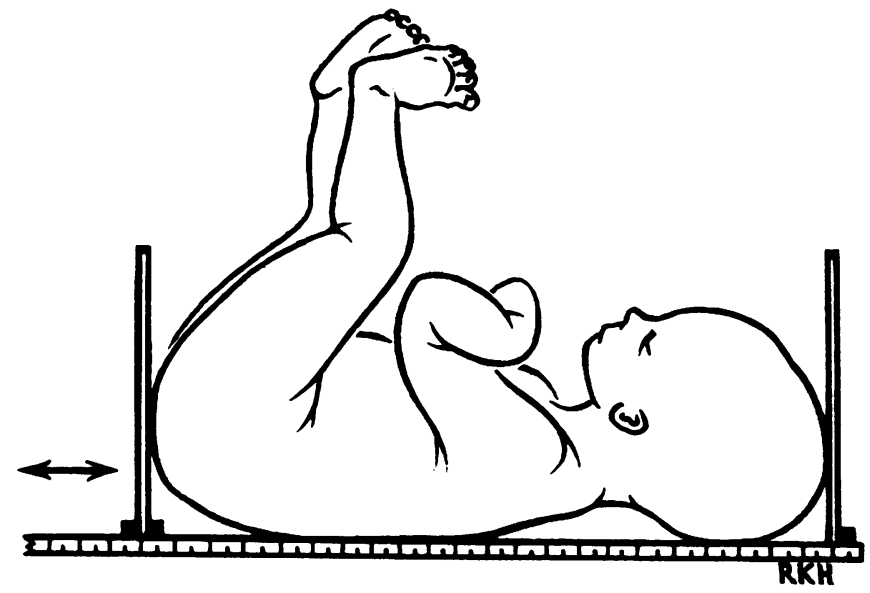

Fig. 2.

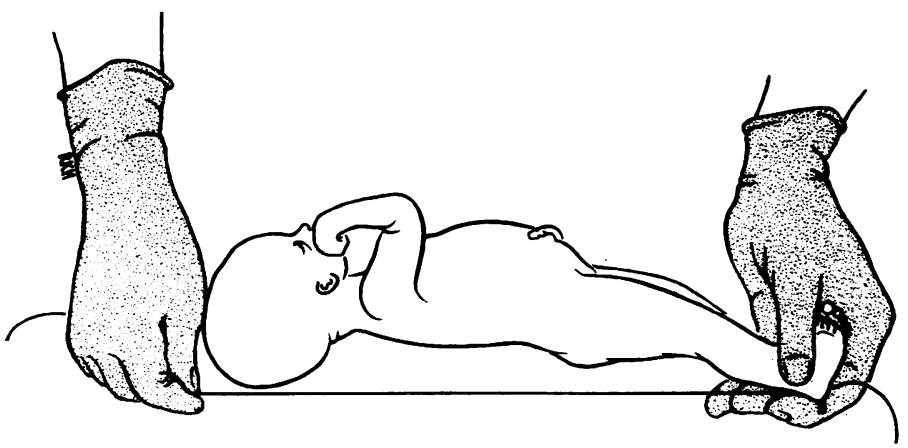

Fig. 3.

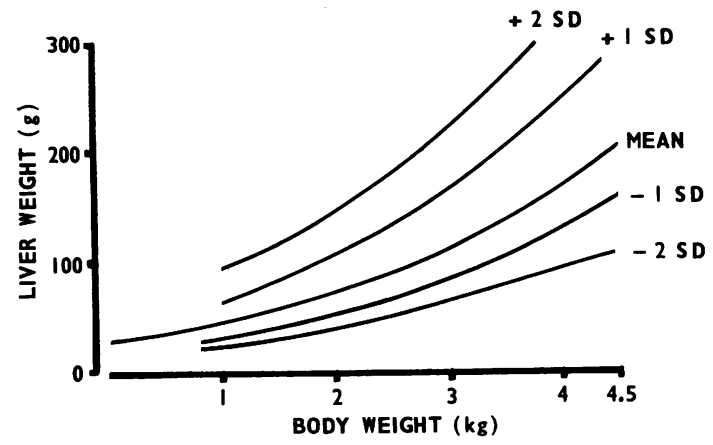
Fig. 1 The crown-heel length is measured by placing
the infant on a graduated board. The head end of the board is fixed but the foot end is movable.

Fig. 2 Measurement of the crown-rump length (sitting height).

Fig. 3 An alternative method of measuring the crownheel length with a thread.

Fig. 4 The variation of liver weight with body weight. The lines marked $+1 \mathrm{SD},+2 \mathrm{SD}$, etc, indicate the contours corresponding to 1 or 2 standard deviations. Using charts like this, or tables, it is possible to determine whether or not an organ is significantly enlarged or unduly small. 
and hydrocephaly. The expected length is very approximately three times the difference between the crown-heel and crown-rump length. These estimates are, however, very crude.

The circumference of the head can be measured with a thread. It is useful in assessing minor degrees of hydrocephaly and microcephaly. The circumference is approximately equal to the sitting height.

The body and organs must be weighed on scales which are both accurate and convenient. Since many foetuses and infants coming to necropsy show retardation of growth it is important to compare the body weight with that of a normal infant of the same maturity. Similarly infants of diabetic mothers and hydrocephalic infants tend to be overweight. Table I, modified from Neligan's table (1965), is useful for making this comparison. Infants whose weight falls more than 2 standard deviations below the mean for the age should be regarded as 'small for dates'. Less severely depressed body weights may need investigation but it must be remembered that any liveborn baby who survives a few days will show a natural fall in body weight for metabolic reasonsparticularly fluid loss.

Potter (1961) gives a table showing the relation between organ weight and body weight but when using such a table it must be remembered that there is some variation about the arithmetic mean as is shown in Figure 4. In his tables of organ weight (brain, head, liver, thymus, and adrenals) Gruenwald (1969) includes the standard deviation. The importance of making these measurements is emphasized when it is remembered that in a small-for-dates baby the brain appears to be relatively large and the thymus relatively small whilst the weights of the heart and liver are unaffected (Gruenwald, 1969).

\section{External Examination}

The external surface of the body is examined for evidence of maceration, cyanosis, petechial haemorrhages, injury, and skin lesions, and the orifices are examined for patency. All abnormalities of development are noted and recorded, especially the rather elusive ones of the face as in Down's syndrome and renal agenesis. It is sometimes useful to supplement these by making a permanent record of the hand and foot prints (Penrose, 1968). The eyes are examined for conjunctival haemorrhages or icterus.

If the foetus has been dead in utero some time, but less than eight hours, the skin often has a parboiled appearance (grade 0 maceration). After this time the skin starts to peel (grade I maceration), and later still peeling may be extensive and red effusions present in the serous cavities (grade II maceration). In prolonged foetal death in utero the liver is yellowish-brown and the effusions turbid (grade III maceration). Sometimes the foetus may appear mummified. Occasionally the skin may peel on an infant who was liveborn but very oedematous.

\section{Examination of the Head and Neck}

One of the major differences in the examination of a baby compared with an adult is in the treatment of the head. This is because the baby's brain is very much softer than that of an adult and damage to the supporting membranes much more common.

The scalp is incised in a line starting at the posterior margin of one ear and extending upward over the top of the head, slightly posterior to the vertex. As the scalp is reflected the amount of oedema and haemorrhage is noted. The width of the sutures and the size of the fontanelles and degree of calcification, eg; craniolacunia, are guides to assessing the presence and degree of hydrocephalus of the skull. In some infants, especially those of African origin, a massive haematoma may cause marked anaemia. Robinson and Rossiter (1968) give the following

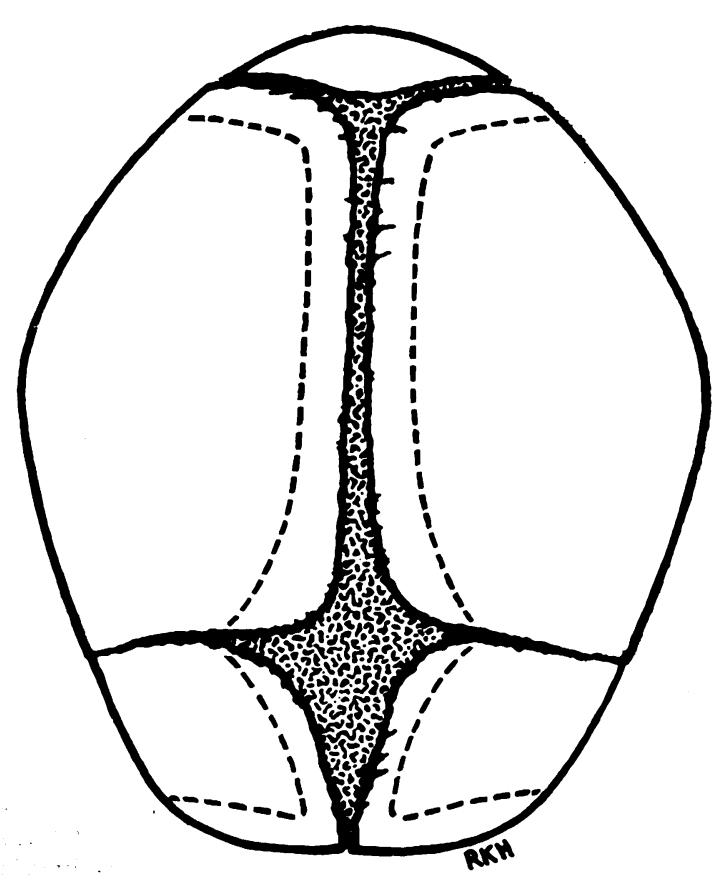

Fig. 5 The interrupted lines indicate the lines of incision when opening the skull. 
formula as a-guide to estimating the volume of the haemorrhage

$$
\mathrm{V}=\left(\mathrm{C}^{3}-\mathrm{C}_{\mathrm{Ex}} \mathrm{x}^{3}\right) / 12 \pi^{2}
$$

where $\mathrm{V}$ is the volume of the haematoma, $\mathrm{C}$ the circumference of the head in centimetres and $\mathbf{C}_{\mathbf{E X}}$ is the expected circumference.

Before opening the vault of the skull the contents of the posterior fossa should be examined by cutting down between the occipital bone and the atlas. Increased pressure of the cerebrospinal fluid can be recognized by the bulging of the meninges through the gap thus exposed and blood or pus can readily be seen when the meninges are incised.

When opening the skull it is important to avoid damaging the meninges and not to spill any blood. To do this the sharp point of the scalpel is inserted near the outer angle of the anterior fontanelle and an incision made with scissors down the coronal suture, including the underlying dura. An incision is then made parallel to the frontal suture but in the bone; at the base of this incision the bone is cut horizontally and a flap of the frontal bone turned back (Fig. 5). The amount of cerebrospinal fluid is noted. In the full-term infant this is negligible and the convolutions lie pressed against the dura; in the premature infant the convolutions are separated from the dura by an appreciable quantity of cerebrospinal fluid. The incision is then continued from its origin at the angle of the fontanelle posteriorly across the upper part of the parietal bone, cutting through the bone a few millimetres from its superior margin. From the posterior superior angle of the parietal bone the incision is directed downwards and forwards, care being taken to cut through bone and not the suture. The superior sagittal and lambdoidal sutures are avoided because the superior sagittal sinus and one portion of the transverse sinus are in direct contact with these structures. The flap of parietal bone is now reflected.

To examine the brain in situ, control of the skull is secured by supporting the infant's head on the palm of the left hand with the chin directed towards the wrist and the neck held between the thumb and fingers. If two fingers of the right hand are gently placed behind the posterior portion of the cerebral hemisphere and the head is tilted laterally and anteriorly, the cerebral hemisphere may be drawn forward, and the tentorium, the falx cerebri, and the vein of Galen may be inspected. The vein of Galen can usually be identified only if it is intact and there is little or no intracranial haemorrhage. After one side of the brain has been examined the bony flaps are replaced to protect the brain and the procedure is repeated on the opposite side. Each cerebral hemisphere may be removed separately by cutting through
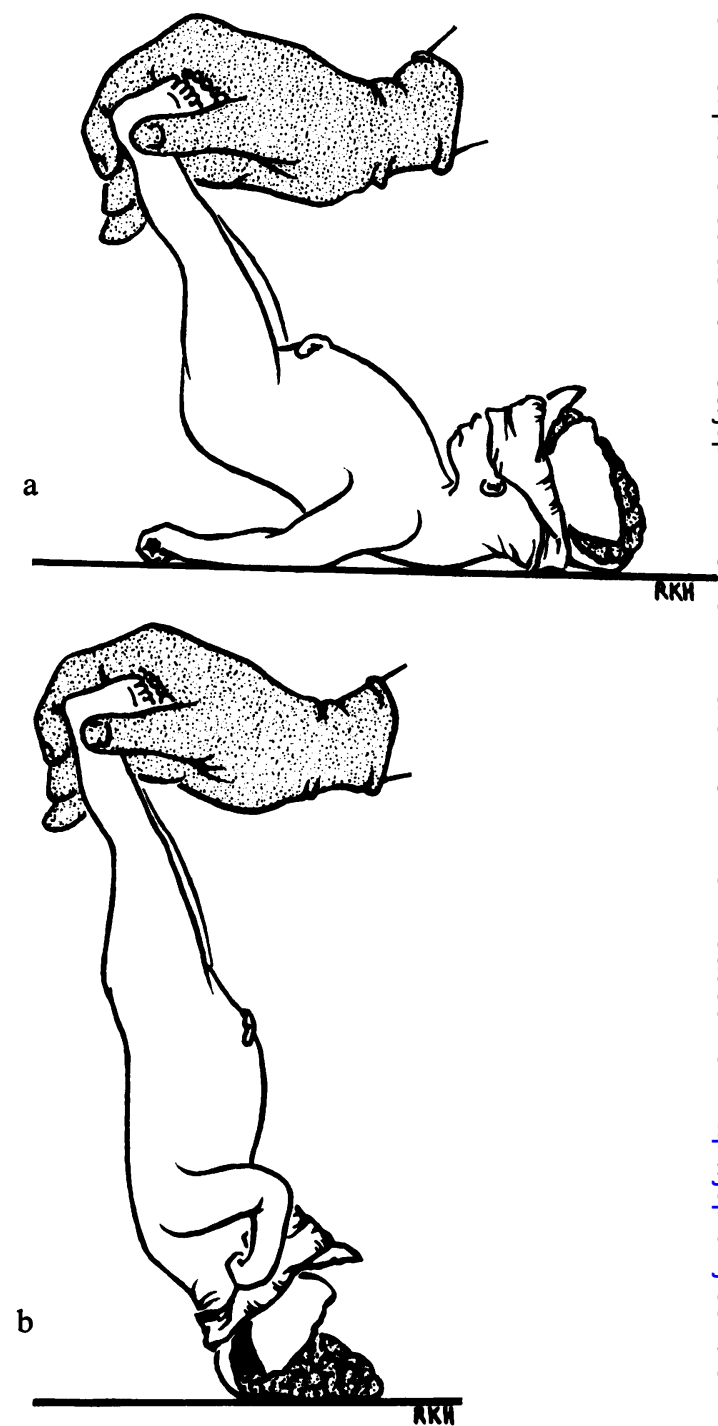

Fig. 6 To avoid damaging the cerebral hemispheres when removing the brain it is convenient to 'pour' the brain out of the skull: $\mathrm{a}$ and $\mathrm{b}$ show two stages of the process.

the cerebral peduncles. Alternatively, the whole cerebrum can be removed by cutting through the mid-brain, after freeing the anterior attachment? of the falx, and then rotating the body feet-over- head, as in Figure 6. This manoeuvre avoids damageo to the soft brain by handling. The pons, medulla and cerebellum may be removed after cutting through $\vec{\nabla}$ the tentorial leaflets and severing the cranial nerves and spinal cord. 
When the brain has been weighed it may be examined immediately by coronal section with a large knife or fixed in formalin for two or three weeks and then sectioned. It is convenient to place the brain on its vertex and cut just in front of the temporal poles, in front of the midbrain, behind the mid-brain, and in the occipital region. Haemorrhage may be present in the lateral ventricles but the congested choroid plexus should not be mistaken for a blood clot. The pons, medulla, and cerebellum are also incised. A more detailed method is outlined in the appendix. When an encephalocele is present it may be desirable to harden the brain before opening the skull. This may be done by injecting saline into the internal carotid artery on each side with a syringe, draining through the jugular veins, and then replacing the saline by formalin; or the cerebrospinal fluid in the lateral ventricles may be replaced by formalin through the anterior fontanelle. The rest of the postmortem examination should then be completed but the dissection of the head deferred for 24 hours. Laurence and Martin (1959) have described an elegant and useful dissection of the skull for cases of hydrocephalus.

The pituitary gland is removed by cutting off the posterior clinoid processes, incising the dura in front of the gland and elevating it with a scalpel from beneath. It is useful to leave a piece of cartilage attached to the gland to act as a marker when it is being fixed with other tissues. In an anencephalic foetus the pituitary gland is often difficult to identify. The base of the skull near the probable site of the gland should then be removed by a saw, decalcified, and the gland looked for microscopically in stepwise blocks.

The venous sinuses should be incised and examined and the middle ears exposed by cutting the petrous bone with bone forceps. After the first week of life it is not uncommon to find a middle ear infection and 'congenital otitis media' has been recorded. When infection is suspected swabs should be examined for pus cells and organisms.

As the brain is examined in situ swabs may be taken from untouched areas. These should be examined both microscopically as well as by culture.

\section{Vertebrae and Spinal Cord}

The vertebral column is examined for fracture and other evidence of injury. The state of the vertebral arteries may be ascertained by histological section (see Appendix). It is important to expose the spinal cord in all cases of hydrocephalus associated with spina bifida to see if an Arnold-Chiari malformation is present. In kernicterus the spinal cord may be involved.
To open the vertebral canal, a middorsal incision is made through the skin and muscles and the tissues dissected away so that the vertebrae are exposed. The laminae are incised with heavy scissors starting either at the foramen magnum or the sacrum and extending along both sides of the spinal processes. The posterior wall of the vertebral column can then be removed. The dura is moderately adherent to the cord. The cord with its covering is removed by cutting through the roots of the nerves.

An alternative and very simple method of opening the vertebral canal is by removing the bodies of the vertebrae. This may be done from the front after the body has been eviscerated.

\section{Mouth and Pharynx}

The mouth, nose, and pharynx should be examined for an obstruction or cleft palate.

\section{Examination of the Thorax and Abdomen}

Usually a longitudinal incision is made from the symphysis pubis to the upper margin of the sternum and continued laterally towards each shoulder. The skin and peritoneum are reflected and the abdominal cavity and anterior thoracic wall exposed. The state of the umbilicus and the number of umbilical arteries is noted. Figure 7 shows an alternative incision which gives very good exposure and demonstrates the state

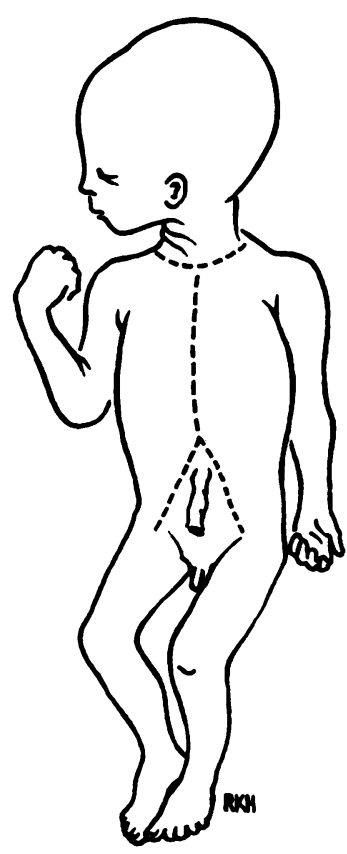

Fig. 7 This shows the lines of incision in the skin when opening the thorax and abdomen. 
and number of the umbilical vessels well. A longitudinal incision is made from the neck to the umbilicus, at which point it divides to form an inverted $\mathrm{V}$, one limb going to the groin on one side and the other to the groin on the opposite side. The umbilical arteries can be seen on the inside of the suprapubic flap as they become the hypogastric arteries and join the iliac arteries.

After inspection of the diaphragm and abdominal viscera strong scissors are used to remove the anterior chest wall. The initial opening of the chest should be under water or saline. I partly fill a sink with water and add a standard quantity of concentrated saline to make the whole approximately isotonic. Other forms of container can be used in lieu of a sink. A simpler method, but less satisfactory if air is loculated in the thorax, is to use a lateral flap of skin to hold a pocket of saline and to open the chest wall through this pocket. A number of infants have interstitial emphysema with pneumothorax and this technique easily reveals this condition. It is useful to make an incision into the heart at this stage while the thorax is under saline; this will reveal the presence of any air in the cardiac blood. This is released much more gradually than the air from a pneumothorax. Intracardiac air may be caused by air embolism following an exchange transfusion or by interstitial emphysema rupturing into the pulmonary vessels. The ribs are cut laterally to the costochondral junctions and the sternoclavicular joint is disarticulated. The organs are inspected in situ and the level of the diaphragm is noted since this gives a measure of pulmonary expansion.

The thymus is removed. This often surrounds the left innominate vein; if it can be dissected from the surface of this vessel without damaging the wall subsequent examination will be easier since bleeding from a large vessel is an annoyance. The pericardium is opened and the positions of the atria, ventricles, great vessels, and ductus arteriosus are noted.

If any abnormality of the heart or great vessels is suspected the thoracic viscera should be removed en bloc together with the upper abdominal organs. Care must be taken to inspect the vessels for congenital anomalies and, if seen, these structures must be carefully preserved for detailed study. In such cases the azygos and bronchial vessels may show abnormalities and occasionally the pulmonary veins may drain into the portal system. Removal of the thoracic viscera in one unit is performed by dissecting the tissues of the neck and tongue and extending the dissection in the posterior mediastinum by cutting behind the oesophagus and aorta.

The oesophagus, larynx, and trachea are opened

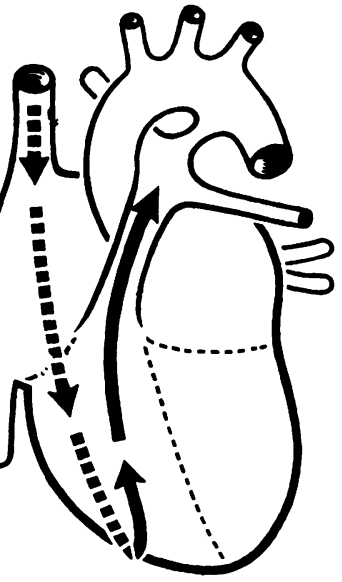

a

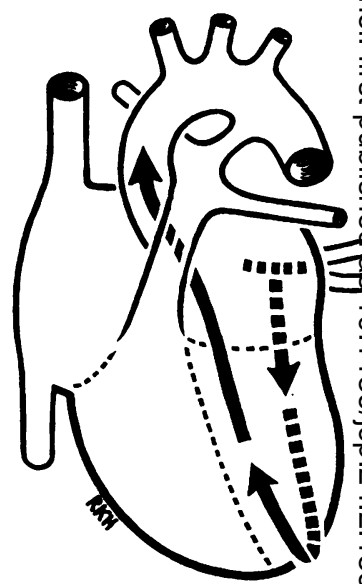

b
Fig. 8 The lines of incision into the right and left sides of the heart are shown when viewed from the anterior surface. The horizontal part of the T-shaped incision in the left atrium joins the orifices of the pulmonary veins.

longitudinally and examined for abnormal contents obstruction, or fistulous openings. Alternativel these may be fixed unopened and examined histo logically without disturbing the mucus plug.

The external and cut surfaces of the lungs are examined. The degree of aeration may be estimate $\overrightarrow{3}$ roughly by flotation but this is crude compare with the information obtained by microscopy. One lung may be set aside temporarily for bacteriog logical examination.

The heart is exposed by removal of the tracheas oesophagus, and lungs. The pericardium is remove $\phi$ by cutting close to the venae cavae, pulmonary vessels, and aorta. The heart is then placed with its posterior surface uppermost and the apex directe away from the dissector. Scissors are inserted into the opening of the superior vena cava and the right atrium and ventricle are then incised lengthwise neas the interatrial and interventricular septa. The tricuspid valve is opened during this procedure. The heart is then turned with the anterior surface upper $\bar{\sigma}$ most and the apex directed towards the dissector. The line of incision is continued round the apex and along the anterior wall to the pulmonary artery an $\$$ along the artery to expose its branches and the ductus arteriosus, if this is still patent. The same procedures is repeated on the left side of the heart, starting fron $\vec{D}$ the pulmonary veins and passing through the mitra? and aortic valves to the aorta (Fig. 8). The septa can be examined and the opening of the coronary arteries 
identified. If the heart is abnormal a drawing should be made for the record. A text such as that of Taussig (1960) or Bremer (1957) is useful as a guide.

\section{The Abdominal Organs}

The examination of the abdomen is similar to that of the adult but the following points should be noted.

1 The liver, stomach, and duodenum, pancreas and spleen are removed en bloc. The tail of the pancreas is removed for histological examination before testing the patency of the common bile duct to avoid damage to the histological appearance by regurgitated bile.

2 The patency of the common bile duct is tested by squeezing the gall bladder and observing the flow of bile into the previously opened duodenum. Only when there is no flow of bile is it necessary to attempt a dissection of the bile ducts.

3 The stomach may be opened to examine for erosions. These are seen most easily by holding the stomach in front of a bright light. In infants who are stillborn or who survive only a few hours the stomach is best fixed unopened, or with only a small incision to allow ingress of fixative, in order that it may be studied microscopically for evidence of swallowed amniotic fluid.

4 The liver is examined by sectioning it coronally, from side to side since the right and left lobes are sometimes different in appearance and demarcated by a sharp line (Emery, 1952).

5 The adrenal is large in the newborn, up to half the weight of the kidney. It diminishes in size absolutely in the first few days after birth. Congestion of the foetal zone may sometimes be mistaken for haemorrhage. In cases of haemolytic disease of the newborn, when the baby is severely jaundiced the adrenal may be bile stained. The cut surface of the adrenal (as in many other small organs) is best exposed, not by cutting with a scalpel, but by drawing a long sharp knife gently over the surface. The weight of the knife will take the blade through the organ without any destructive manual pressure.

6 Foetal lobulation of the kidney is normal at this age.

7 In the male if the testes are not in the scrotum or inguinal canals examine the peritoneal cavity for an abdominal site.

8 By cutting through the symphysis pubis the prostate with the bladder in the male and the whole of the genital tract in the female can be removed, together with the kidneys and adrenals and abdominal aorta. The ureter should be visualized both in situ and after removal with the bladder and kidneys.

\section{Examination of the Skeleton}

A modern necropsy suite includes $x$-ray apparatus (Association of Clinical Pathologists, 1961). When such apparatus is available it is useful to have an $x$-ray photograph of each baby coming to necropsy. Although the film may not be ready at the time of the postmortem examination the $x$-ray plate often gives a better illustration of a bone abnormality than a verbal description. If the film is ready it may guide the pathologist. For example, a fistulous communication through a vertebral body between a thoracic cyst and the spinal cord can be seen, or unsuspected ectopic calcification recognized. $X$-ray films may also be used for determining the age of the foetus.

In the absence of any indication to examine particular parts of the skeleton the femur should be taken as a typical example. A horizontal incision is made across the knee below the patella and extended into the joint cavity after which the skin and muscles are dissected from the lower half of the femur. The lower half of the femur is removed with bone forceps and then cut longitudinally. A centre of ossification in the cartilage at the lower end of the bone is usually visible about the beginning of the 38th week of gestation and is evidence of foetal maturity. The epiphyseal line is usually straight and has no measurable width. In syphilis, and sometimes in rare types of chondrodystrophy, an irregular zone 1 to $2 \mathrm{~mm}$ in width is present; in the absence of generalized skeletal malformations this usually indicates syphilis. The upper end of the tibia, the os calcis, talus, and tarsal cuboid are examined for ossification centres to assess maturity. The ossification centres in the sternum can also be used for this purpose; they can be seen by transillumination or by splitting the sternum with a knife.

\section{The Placenta}

No foetal necropsy is complete until the placenta has been examined both macroscopically and microscopically. Disease of the decidual vessels and extensive infarction may be important factors in causing foetal death in utero when the mother suffers from toxaemia of pregnancy. Inflammation of the placenta and membranes is always present in foetal pneumonia (syndrome of the infected amnion).

The umbilical cord should be inspected for knots, ruptured varices, number of arteries, and its site of insertion noted, whether into the placenta or membranes. At term the placenta is approximately oneseventh of the foetal weight but in syphilis and haemolytic disease and certain other conditions the placenta may be much enlarged; on the other 
hand, if the placenta is much smaller than expected placental insufficiency may be considered as a contributing factor in death. The colour of the foetal surface of the placenta should be recorded. If there has been foetal distress it is often green because of the uptake of bile from excreted meconium. The presence of haematomas or tumours should be noted. The maternal surface should be examined for calcification or for evidence of compression caused by blood clots following premature separation of the placenta. Yellow, or buff-coloured, areas on the maternal surface may sometimes be caused by infarcts but other common lesions, such as perivillous fibrin deposition, may cause them.

In order to assess the type and extent of the lesions the placenta should be fixed for about a week before sectioning. It should then be cut in serial slices $\frac{1}{2}$ to $1 \mathrm{~cm}$ thick either with a long, very sharp knife or on a bacon slicer. A central slice,

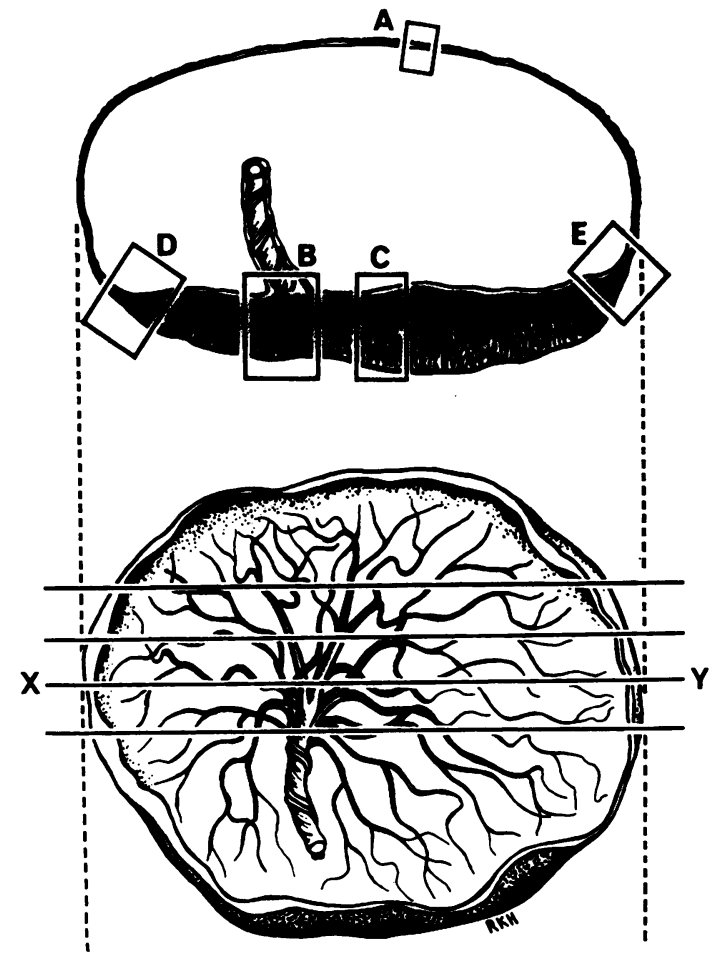

Fig. 9 The placenta is cut into a number of thin slices parallel to a line (XY) through the insertion of the umbilical cord. Blocks of tissue for microsopic examination are taken routinely from the central slice. A, from the torn margin of the membranes; $\mathrm{B}$, from the site of insertion of the umbilical cord; $\mathrm{C}$, a central block if the cord is inserted eccentrically; D and $\mathrm{E}$, from each margin of the central slice to include both placental tissue and the adjacent chorionic membrane. running along a diameter and including the insertion of the umbilical cord, is removed for routine histo logical sections. Prolonged fixation makes the tissue firm and provides a better cut surface than section-? ing after short fixation, when the tissue will stilf be friable. The various lesions that may easily be seen include true infarcts, perivillous fibrin deposits intervillous thrombosis, and septal cysts (Fox 1963, 1964; Devi, Jennison, and Langley, 1968) Four or five blocks of tissue should be taken fromb the central slice and membranes for microscopie examination: one through the whole thickness of the placenta at the centre of the slice and another af the site of insertion of the cord if this is not centralo one at each end of the central slice and including a little of the adjacent membrane; another in the membranes at the site of rupture; and a transverser section of the cord about an inch from the foetaf surface (Fig. 9). These blocks are important in assessing the mode of spread and extent of inflam $\vec{z}$ mation when it is present. Blocks should also bळ taken from representative lesions; the nature of many so-called 'white lesions' can only be determined by microscopic examination. When the placent\& is from twins and a septum exists between the amniotic sacs this should be examined histologically to determine the presence or absence of chorion.

\section{Tissue for Microscopy}

If the body is that of a macerated stillborn infant it is generally not profitable to examine many tissues histologically. It is important to examine the plar centa of such an infant, a piece of liver to provide objective evidence of the degree of maceration, and representative sections of the lung. The lung may show much inhaled liquor amnii, indicative of intrauterine hypoxia, or erythroblasts, suggestive of haemolytic disease.

If the body is not macerated the minimum number of blocks that should be taken in each case is $(B)$ each lung, if possible whole coronal sections, or block from each lobe; (2) right and left lobes of the liver; (3) pancreas, the islets may show significant changes in infants of diabetic mothers, in babies with haemolytic disease, and occasionally in othep diseases. (4) kidney, useful in assessing the degree of maturity; (5) oesophagus, one block from the post-cricoid region where loss of epithelium mag be seen after intubation and another from the lowert end where ciliated epithelium may be seen to :a varying extent depending on the maturity of the infant; and (6) the placenta, as described above.

Abnormalities in other organs will naturally suggest further blocks which should be taken.

Under ideal conditions the following tissues shoukd 
also be taken but their examination by an inexperienced person may be misleading:

\section{ALIMENTARY TRACT}

(1) the parotid gland to detect cytomegalic inclusion disease if this is endemic; (2) the tongue; (3) the stomach, eg, for swallowed amniotic fluid; (4) the intestine, if indicated.

\section{CARDIOVASCULAR SYSTEM}

Sections of the two ventricles to indicate their relative thickness.

\section{UROGENITAL SYSTEM}

(1) Bladder, (2) prostate, (3) testis, (4) ovary, (5) uterus with cervix,(6) vagina. The prostate and vagina give some indication of the effect of circulating maternal steroid hormones.

\section{LYMPHOID TISSUE}

(1) The thymus, (2) the mesenteric lymph nodes,

(3) the spleen.

\section{ENDOCRINE ORGANS}

(1) Pituitary, (2) thyroid, (3) adrenal. Fat staining (sudan IV) is usually necessary for the interpretation of changes in the adrenal (Langley and Burne, 1955; Burne and Langley, 1956).

BRAIN

See Appendix

\section{THE UMBILICUS}

The adjacent abdominal skin should be included as when the cord has separated there may be a mild inflammatory reaction.

\section{BONES}

(1) The lower end of the femur, (2) the fifth rib to include the costochondral junction (Emery and Kalpaktsoglou, 1967).

\section{FIXATION}

It is important that the fixative should be adequate in volume-at least 20 times the volume of tissue. In practice this means putting fairly small pieces of tissue into a vessel containing about a litre of fluid. Formalin is the most convenient primary fixative, especially if histological stains for lipids and other substances are to be used. Ten per cent formalin neutralized by standing over calcium carbonate or buffered formalin may be used. Formalin may form a pigment with haemoglobin which may obscure structural details but this pigment can be removed by potassium hydroxide. Postfixation with formolsublimate for a few hours before processing improves the quality of the staining. Special stains may give valuable information especially fat stain (lung, liver, adrenal, heart, and kidney) and stains for glycogen (heart, liver). However, the interpretation of the findings requires some experience of perinatal pathology.

\section{Specimens for Bacteriological Examination}

BLOOD

Blood fur culture may be obtained from the heart using a sterile dry syringe or Pasteur pipette after first searing the surface with a soldering iron or some such instrument. Blood may similarly be obtained from the anterior fontanelle. If the blood is not haemolysed it may also be useful for serological tests.

\section{LUNG}

One lung is removed and placed into a dry container. In the laboratory the surface of the lower lobe is dried and seared. An incision is made through the seared area and a stiff platinum wire with a large loop, or punch forceps, is passed into the lung tissue probing in several directions. It is often possible to remove a piece of tissue by this means but the consistency of the lung may prevent this. The material obtained is cultured aerobically and anaerobically on blood agar plates and in Brewer's medium and some may be sent for virological examination.

\section{PLACENTA}

The most useful specimen is blood obtained from the veins on the foetal surface of the placenta. After wiping and searing the membranes a Pasteur pipette is passed through into the vessel and as large a specimen of blood as possible is obtained and inoculated into Brewer's medium. The tissue of the placenta can also be examined by a similar technique to that used on the lung passing the loop or punch forceps through an area of seared membranes.

\section{Correlation of Postmortem Findings}

The postmortem examination itself does not usually give more than the immediate cause of death and quite often this may not be obvious. It is necessary to correlate the naked-eye, histological, and microbiological findings with the clinical observations on both the mother and baby before the sequence of events leading to the death of the infant can be traced. It is only by understanding this sequence that perinatal mortality can be reduced. 
in the fixative. Fifteen per cent formol saline is recommended as a fixative and fixation should -

\section{Appendix}

The following further methods of examination are useful but not necessary for routine use.

\section{Examination of the Cervical Vertebrae and Vertebral Arteries}

Yates (1959) has shown that there may be lesions of the vertebral vessels such as thrombosis or perivascular haemorrhage. The cervical vertebrae should be removed en bloc, fixed, decalcified, and transverse sections cut at three levels. In the presence of hydrocephalus this technique must be modified. The laminae must then be removed to expose the cervical cord.

\section{Examination of the Brain}

The following method is based on suggestions made by Gruenwald and Laurence (1968).

Weigh the brain after removal either before placing it in the fixative, or, if the weight of the jar with fixative is known, the brain may be weighed

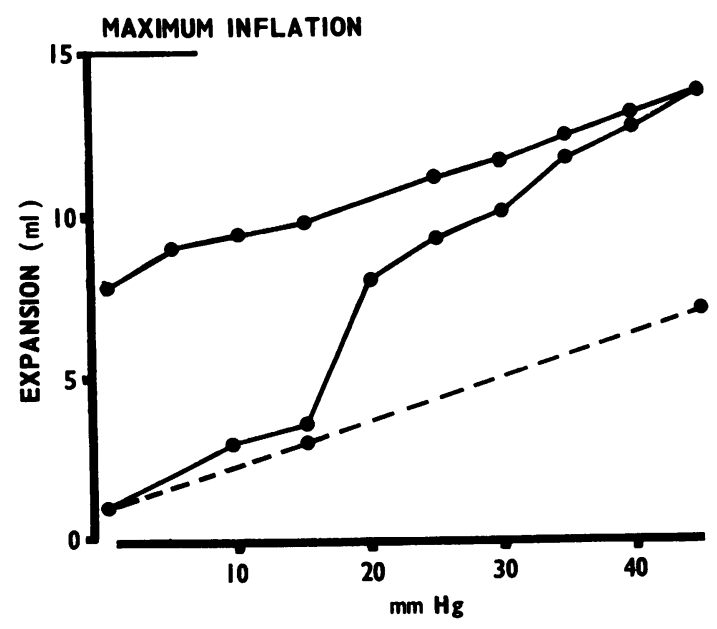
last two or three weeks. When the brain is tolerably firm it should be placed on a cutting board with the ventral surface upwards and be cut in a ventro $\frac{-}{5}$ dorsal direction at the level of the mamillary bodiese The anterior and posterior halves should then b. cut in a series of slices $0.5-1.0 \mathrm{~cm}$ thick parallel to this initial incision.

Blocks should be cut from the following sites: $(1 \vec{D}$ the insula, the inferior temporal sulcus, Ammon's horm and the adjoining part of the temporal lobe; (2) the globus pallidus, thalamus, and subthalamic nucleis (3) the middle part of the superior frontal gyrus which includes the boundary zone of the areass supplied by the anterior and middle cerebral arteries (4) the centrum semiovale adjacent to and including the wall of the posterior horn of the lateral ventricle (5) a block at right angles to the calcarine sulcus? to include the calcarine cortex; (6) a slice from the cerebellum to include the dentate nucleus; (7) a slicen from the upper end of the pons; (8) a slice from the medulla at the level of the inferior olives.

These blocks are embedded in paraffin and sections stained with haematoxylin and eosin and with phosphotungstic acid haematoxylin or one of its variants. The former will show ganglion cells, blood

Fig. 10 An inflation curve of an excised 'normal' lung from an infant dying at 8 hours with a tentorial tear and intracranial haemorrhage. The lower continuous line is the pressure-volume curve during inflation, the upper continuous line is the pressure-volume curve during deflation. The deflation curve does not return to the origin, the lung remains inflated. The interrupted line is the pressure-volume curve of the apparatus without the lung.

Fig. 11 An inflation curve of an excised lung of an infant dying with pulmonary hyaline membranes. The infant died six hours after delivery by Caesarean section. The upper deflation part of the curve returns to the origin; the lung does not remain inflated because of the lack of surfactant. 
vessels and meninges and the latter will show gliosis, myelin sheaths, and fibrin. It is advantagous, but not essential, to cut frozen sections of all blocks for fat staining to show up degeneration of ganglion cells and white matter.

Anoxic degeneration of ganglion cells may be seen in the thalamus, hippocampus, nuclei of the hind brain, and Purkinje cells of the cerebellum. Anoxic changes have a rather similar distribution to those of bilirubin encephalopathy. Other changes may occur at the borders of zones supplied by different arteries (Norman, Urich, and McMenemey, 1957). Such a 'watershed' area is included in block 3. In premature infants subependymal haemorrhage is common over the caudate nucleus (block 2). The spinal cord should always be examined.

\section{Examination of Tissue for Viruses}

For preference the tissue should be inoculated into medium as soon as possible and sent to the virus laboratory immediately. The tissue may be kept at $4^{\circ} \mathrm{C}$ but it should not be deep frozen.

\section{The Lungs}

It is an advantage to fix one of the lungs in a distended state. This may profitably be combined with measuring the changes in volume of the lung with pressure. These changes have been discussed by a number of workers (Avery, 1968) and Figs. 10 and 11 show the differences between a 'normal lung' and one with pulmonary hyaline membrane.

I should like to thank Dr G. Neligan and the Spastics Society Medical Education and Information Unit for permission to publish Table I, and Dr R. Ollerenshaw and Mr R. K. Harrison, of the Department of Medical Illustration, United Manchester Hospitals, for the diagrams.
References

Association of Clinical Pathologists (1961). Mortuary design and hazards (Report of Committee). J. clin. Path., 14, 103-108.

Avery, M. E. (1968). The Lung and its Disorders in the Newborn Infant; 2nd ed., pp. 158-162. Saunders, Philadelphia.

Bremer, J. L. (1957). Congenital Anomalies of the Viscera. Harvard University Press, Cambridge, Mass.

Burne, J. C., and Langley, F. A. (1956). The changes in the adrenal cortex in haemolytic disease of the newborn. J. Path. Bact., 72, 47-57.

Devi, B., Jennison, R. F., and Langley, F. A. (1968). Significance of placental pathology in transplacental haemorrhage. J. clin. Path., 21, 322-331.

Emery, J. L. (1952). Degenerative changes in the left lobe of the liver in the newborn. Arch. Dis. Childh., 27, 558-561.

Emery, J. L., and Kalpaktsoglou, P. K. (1967). The costochondral junction during later stages of intrauterine life and abnormal growth patterns found in association with perinatal death. Arch. Dis. Childh., 42, 1-13.

Fox, H. (1963). White infarcts in the placenta. J. Obstet. Gynec. Brit. Cwlth., 70, 980-991.

Fox, H. (1964). Calcification in the placenta. J. Obstet. Gynec. Brit. Cwlth., 71, 759-765.

Gruenwald, P. (1969). Growth and maturation of the foetus and its relationship to perinatal mortality. Chapter 8 of In Perinatal Problems, edited by N. R. Butler, and E. D. Alberman, ch. 8, pp. 145-162. Livingstone, Edinburgh.

Gruenwald, P., and Laurence, K. M. (1968). A method of examining the brain of the newborn. Develop. Med. Child. Neurol. 10, 64-68.

Langley, F. A., and Burne, J. C. (1955). The effect of disease and injury on the adrenal cortex of stillborn and newborn infants. Arch. Dis. Childh., 30, 141-146.

Laurence, K. M., and Martin, D. (1959). A technique for obtaining undistorted specimens of the central nervous system. J. clin. Path., 12, 188-190.

Neligan, G. (1965). A community study of the relationship between birth weight and gestational age. In Gestational Age, Size and Maturity (Clinics in Developmental Medicine, No. 19), edited by M. Dawkins, and W. G. MacGregor, pp. 28-32. Heineman, London.

Norman, R. M., Urich, H., and McMenemy, W. H. (1957). Vascular mechanisms of birth injury. Brain, 80, 49-58.

Penrose, L. S. (1968). Medical significance of finger-prints and related phenomena. Brit. med. J., 2, 321-325.

Potter, E. L. (1961). Pathology of the Fetus and Infant, 2nd ed. Yearbook Medical Publishers, Chicago.

Robinson, R. J., and Rossiter, M. A. (1968). Massive subaponeurotic haemorrhage in babies of African origin. Arch. Dis. Childh., 43, 684-687.

Taussig, H. B. (1960). Congenital Malformations of the Heart, 2nd ed., Commonwealth Fund, Harvard University Press, Cam. bridge, Mass.

Yates, P. O. (1959). Birth trauma to the vertebral arteries. Arch. Dis. Childh., 34, 436-441. 
to be regretted that no index of names is included, but there can be no doubt at all that, like its predecessors, this work is of the greatest value to all interested in cardiac pathology.

T. CRAWFORD

Verhandlungen der Deutschen Gesellschaft für Pathologie By G. Seifert. (Pp. 613; illustrated. No price.) Stuttgart: Gustav Fischer Verlag. 1969.

Cardiovascular Pathology By R. E. B. Hudson. Vol. 3. (Pp. $x-1,166$; illustrated. £24) London: Edward Arnold. 1970.

In our notice of volumes 1 and 2 we described Professor Hudson's book as an astonishing magnum opus: the addition of volume 3 just five years later leads us to the verge of incredulity! We gently chided Professor Hudson for adding to the enormous length of his work by including such things as postmortem room technique, photographic technique, and detailed accounts of quite common microorganisms; but the spots have not changed and the new volume includes accounts of the ultrastructure of living matter, the genetic code, and the classification of proteins. This attitude contributes significantly to the size and cost of the three volumes and frankly these marginally relevant subjects would be better studied in the many specialized monographs available.

The new volume is described as a 'supplement' to the former work and has been produced as the alternative to a complete second edition. We congratulate the author and publishers on this arrangement, for, though the cost is high, it is clearly less than that of a new edition would have been. Furthermore it has enabled quicker publication with the result that the new volume is much more up to date than most large volumes are by the time they are in the reader's hands.

The contents of the new volume are arranged in 43 chapters corresponding to those of the original volumes. In each a comprehensive review of recent work on the subject is given and references to the pages in the earlier volumes are provided. The result is a thoroughly up-to-date presentation at the reader's disposal. The author suggests that information on a required subject should first be sought in the new volume.

As one of the main uses of these volumes will be as a source of references it is
This book is an account of the 53rd meeting of the German Society of Pathology, in which the main theme was intestinal malabsorption. The book is beautifully printed and illustrated and every chapter begins with a summary in English. This volume should be read by those having a special interest in the pathology of intestinal malabsorption but there are also interesting articles in the field of experimental pathology and cancer research. The papers on pancreatitis and lesions of the liver, experimental aspects of hepatitis, and the pathology of atherosclerosis are particularly outstanding. The papers on malabsorption includes an excellent review on morphology and pathogenesis as well as the importance of epithelial regeneration and differentiation in the normal and abnormal function of the small bowel. There are also well presented articles on the biochemistry and histochemistry of the small intestine in malabsorption and an outstanding paper on the physiology and pathology of mucus formation in the intestine.

For those pathologists speaking the German language the book is strongly recommended but for those who do not speak it the summaries in English together the content.

B. MORSON

Tumours of the Urinary Bladder By K. P. Sarma. (Pp. 443; 25 tables. £5.) London: Butterworths and Co. Ltd. 1969.

The scope of this book is wider than the title suggests and many parts will be of greater value to clinicians than to pathologists. There is much to criticize in the way some of the pathological processes are classified and that the esoteric trends are overemphasized at the expense of the ordinary. It is a great pity the account of the UICC classification of epithelial tumours, which many hope will have wide acceptance, is so inadequate. There are with the illustrations give a good idea of several obvious inaccuracies, and irritating discrepancies between tables and their related texts. Despite these shortcomings, the book can be recommended as a useful source of up-to-date references.

R. C. B. PUGH

\section{Notices}

\section{The International Society on Thrombosis} and Haemostasis

The second meeting of the International Society on Thrombosis and Haemostasis will be held in Oslo, Norway, from 12 to 17 July 1971 . The scientific sessions will include papers on the main topics, free communications of original research, and small group discussions. Participation is open to all scientists interested.

For information concerning membership write to James M. Stengle, M.D., Secretary-Treasurer, International Society on Thrombosis and Haemostasis, National Heart and Lung Institute, Bethesda, Maryland 20014, USA, and for all matters pertaining to the congress to the Secretariat, Second Conference of the International Society on Thrombosis and Haemostasis, Institute for Thrombosis Research, Rikshospitalet, Oslo, Norway.

European Division of the International Society of Haematology

The first meeting of the European Division of the International Society of Haematology will be held from 10 to 12 September, 1971 in Milan (Italy). The main subjects will be the haemolytic anaemias and the lymphocytes.

For further information, registration, and abstract forms, please contact A. T. Maiolo, Secretary of the First Meeting of the European Division of ISH, Istituto di Patologia Medica. Via Pace 15 20122, Milano (Italy).

\section{Price Correction}

The price of Reprint No. 2, 'The perinatal postmortem examination', by F. A. Langley published in March $(J$. clin. Path., 1971, 24, 159) is incorrectly stated in the footnote. The price is $25 \mathrm{p}$, and the reprint can be obtained from the Publishing Manager, BMA House, Tavistock Square, London WC1H 9JR, the envelope marked, Journal of Clinical Pathology, BROADSHEETS. 